\title{
تطبيقات البرمجه الخطية باستخدام طريقة السمبلكس ذات المرحلتين
}

أسماء عمر علي امبيرش1، زينب العربي الكواش²، سعاد مولود زلي3

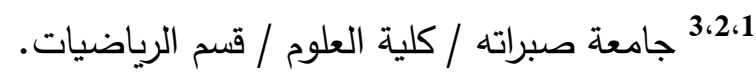

1 asmaomar339.@gmail.com

الملخص

نبحث في هذه الورقة تطبيقات البرمجة الخطية في نماذج النقل وخصوصا عند وجود مشاكل للإنتاج الأمثل؛ حيث قمنا بمقارنة بين الطرق الحديثة للوصول إلى تعظيم الأرباح باستخدام البرمجة الخطية الحديثة ومعرفة كيفية تحويل المدخلات المتاحة إلى معادلات رياضية يمكن حلها بسهولة، ويتم ذلك من خلال بناء نموذج رياضي لمشكلة البرمجة الخطية حيث تعجز طرق الحل الاعتيادية عن حل نموذج البرمجة لعدة مراحل. وقد تم استخدام برنامج LINDO و وبرنامج WINQSB ومقارنته بطريقة السمبلكس ذات المرحلتين للوصول للحل الامثل. وتم التطبيق في شركة مرسى زواغة لتعليب الأسماك في

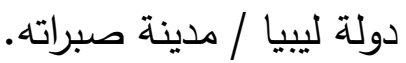

الكلمات الرئيسية: طريقة السمبلكس ذات المرحلتين، برنامج LINDO، برنامج WINQSB.

المقدمة

تأسّست الشركة سنة 2005 وهي من أهم الشركات لإنتاج التونة والسردين، والتي تقوم بتوفير السردين من السوق المحلى ومن الصيادين من شاطئ البحر داخل البلاد، أما التونة فتقوم باستيرادها من الخارج من شرق آسيا وسلطنة عمان عن طريق السفن. تتحمل الثركة تكلفة النقل للبضائع والمنتوجات من المخازن الموجودة داخل الثركة إلى مراكز التوزيع (التي تعد المحطة النهائية للتونة والسردين). 
ويدخل في احتياجات الثركة الثلج المجروش لتبريد الأسماك ويكون متوفر على طول السنة محليا ويتبع الأسماك حسب طلب السلعة وحسب الإمكانيات لذلك، وأيضا زيت الزيتون من الإنتاج المحلي داخل البلاد.

ومن خلال المتابعة الفاعلة والمستمرة لغرض التحقق من تتفيذ الخطة التي تم وضعها عن طريق ضبط وتوجيه أنشطة العمليات خلال العملية الإنتاجية من بدء لحظة وصول الخامات وبدء العمليه الإنتاجيه حتى مراحل التسليم النهائي للمنتج أو الخدمة للمستهلك، ومن خلال ذلك يسعى الباحثون لتحقيق أهداف الشركة وبكفاءة عالية باستخدام الأساليب العلمية من أهمها في هذا المجال البرمجة الخطية عن طريق استخدام النماذج الرياضية بشكل واسع والتخطيط للإنتاج والوصول بالثركة للأمثليه المرجوة.

في هذا البحث قمنا بتحويل النموذج الرياضي إلى نموذج برمجة خطية حيث يتم إيجاد الحل الأمثل باستخدام برنامج LINDO و برنامجWINQSB ومن ثم يتم مقارنته بالحل الجبري (الطريقة المبسطة ذات المرحلتين) للوصول إلى صافي الربح للسلع المنتجة.

مقدمة على البرمجة الخطية (Linear Programming)

تعدّ البرمجة الخطية من أهم أساليب البرمجة الرياضية وأكثرها تطبيقا في الحياة العملية لضمان الاستخدام الأمثل للموارد المحدودة في ظل الإمكانيات المحدودة .مثل إيجاد المزيج الأمثل من بين المنتجات التي ينتجها مصنع معين لتحقيق أكبر ربح طبقا للمتاح من العمل والمواد الخام .وكنلك مثل نقل منتجات معينة من مناطق إنتاج إلى مراكز استهلاك بحيث تقوم كل منطقة إنتاجية بتوزيع منتجاتها إلى مراكز الاستهلاك بحيث يشبع كل مركز استهلاكي طلبه بأقل تكلفة ممكنة .

فأسلوب البرمجة الخطية يستخدم في حل المشاكل المتعلقة بتخصيص الموارد النادرة من الاستخدامات البديلة المتاحة في أفضل تخصيص بهدف تعظيم دالة منفعة متخذ القرار وذلك بتخصيص الموارد المتاحة بصورة تحقيق أقصى أرباح ممكنة إذا كان الهدف تعظيم الربح (profit maximization) أو تذنية الكلفة إذا كان الهدف هو تقليل الكلفة (cost maximization) وأغلب مشاكل النقل يتم صياغتها بواسطة نموذج برمجة خطية على أساس تقليل كلفة النقل. 


\section{شروط البرمجة الخطية}

1. القدرة على تحديد المشكلة موضوع البحث تحديدا رياضيا دقيقا.

2. محدودية الموارد البشرية والمادية الخاضعة للبرمجة مثل محدودية رأس المال، وعدد العمال، والبضاعة المستوردة، والطاقة الإنتاجية وغيرها. 3. أن تكون العلاقة بين المتغيرات هي علاقة خطية. 4. توفر استخدامات تنافسية للموارد البشرية والمادية موضوع البرمجة الخطية مثلا إنتاج منشأة سلعتين. - (- n 5. إمكانية التعبير عن الفعاليات والمتغيرات لموضوع البرمجة بصورة رقميه. (النجار ظافر حسين النجار ، صباح كريم القيسي، ثائر فيصل. (2009)). مكونات النموذج الرياضي Decision variables and parameters متغيرات القرار والمؤشرات ويمكن تعريف المتغيرات على أنها هي الكميات غير المعروفة التي يحدها الحل وتخضع لإرادة متخذ القرار مثل تحديد الكميات المطلوب إنتاجها من منتجات مختلفة ينتجها المصنع أو تحديد الكميات المطلوب نقلها من المصانع إلى الأسواق. بينما الثوابت أو المؤشرات فيمكن تعريفها بأنها هي الكميات المعروفة الثابتة التي بناء عليها يتم تحديد المتغيرات مثل الكميات المتاحة من كل مورد أو الكمية المستخدمة من مورد معين لإنتاج وحدة واحدة من منتج ما أو معدل الربح أو تكلفة منتج

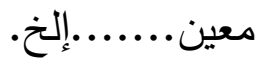
دالة الهدف Objective Function تعتمد على مجموعة من المتغيرات. القيود Constraints مجموعة من القيم يتم فرضها على المتغيرات أو بعض المتغيرات. إن الصيغة الرياضية لنموذج البرمجة الخطية هي كالآتي: Optimise $\sum_{J} C_{J} x_{J}$ Minimise) or (Maximise 


$$
\begin{gathered}
\text { subject to } i=1,2, \ldots ., m \quad b_{i} \sum \sum a_{i j} x_{j}\left\{\begin{array}{l}
\geq \\
= \\
\leq
\end{array}\right\} \\
x_{J} \geq 0 \quad J=1,2, \ldots ., n
\end{gathered}
$$$$
\text { إذ أن }
$$$$
\text { • }
$$$$
\text { bi }
$$

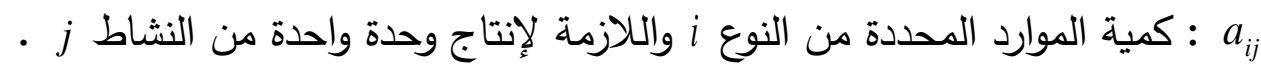
شرط عدم السلبية (...... ش $x_{i}>=0(i=1,2$ (د. حميد ناصر الفتال، د. دلال صادق الجواد، (2008م)). هناك ثلاث طرق رئيسية تستخدم لحل مشاكل البرمجة الخطية وهي:

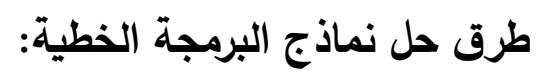

1. مريقة الرسم البياني The Graphical Method. 2. Algebraic Method مريقة الجبرية

3. The Simplex Method طريقة الصف البسيط السمبلكس

طريقة الرسم البياني (Graphic Method) : تستخدم هذه الطريقة لحل مشاكل البرامج الخطية التي لا يزيد عدد المتغيرات فيها عن متغيرين ويمكن حلها بصعوبة في حال ثلاث متغيرات. ويتعذر رسم النموذج في حال إحتوائه على أكثر من ثلاث متغيرات. ولحل المشكلة باستخدام هذه الطريقة يتم التعبير عن كل منتج أو نثاط بأحد الإحداثيات. وهذه الطريقة عبارة عن رسم بياني لنموذج البرمجة الخطية ويتكون النموذج البياني من الإحداثي الأفقي والإحداثي الرأسي (العمودي). كما أن هذه الطريقة تقوم على فكرة تمثيل القيود بمعادلة خط مستقيم وبعد ذلك ترسم القيود المفروضة وبذلك نحدد المنطقة أو الحيز 
الممكن Feasible Region أو منطقة الحلول الممكنة، ثم يتم تحديد أفضل نقطة في منطقة الإمكانيات لتكون الحل الأمثل. ( د. محمد محمد كعبور ، (2005م)).

طريقة الحل الجبري (The Algebra Method) : وهي تمثل أسلوبا آخر من أساليب البرمجة الخطية وهذه الطريقة تتميز باتساع استخدامها في حال زيادة عدد المتغيرات عن اثثين.

الطريقة المبسطة (Simplex Method): في حال وجود أكثر من ثلاث متغيرات في المشكلة فإنه لا يمكن استخدام الطريقة البيانية وإنما علينا استخدام طريقة أخرى مثل الطريقة المسماة بالطريقة المبسطة Simplex Method اختياري تكراري لتحليل مشاكل البرمجة الخطية ويعتمد هذا الأسلوب على اختيار المتغيرات ذات التأثير الأساسي على كلٍ من دالة الهدف والقيود أي المتغيرات التي تؤدي لتحسين قيمة دالة الهدف ويهمل المتغيرات الأخرى التي لا تؤثر على دالة الهدف والقيود . (Hillier, T., Liberman, J., (2005). كما تعرف الطريقة المبسطة بأنها وسيلة رياضية ذات كفاءة عالية في استخراج الحلول المثلى لمشكلات البرمجة الخطية بصورة عامة. وبسبب إمكانية برمجة المعلومات لمشكلات البرمجة الخطية على الحاسبة الآكترونية بهذه الطريقة أدى ذلك إلى انتشار استخدام هذه الطريقة على مدى واسع وبصورة كبيرة، ولذلك استخدمنا الطريقة المستحدثه منها وهيا طريقة السمبلكس ذات المرحلتين في هذا البحث.

نموذج النقل (Transportation Model)

يِّد نموذج النقل أحد النماذج الرياضية الخاصة والذي يهدف إلى إيجاد أسلوب أمثل لتوزيع (نقل أو شحن) تقوم فكرة نماذج النقل على أساس النقل الاقتصادي للوحدات الإنتاجية المتجانسة من مصادر الإنتاج أو التسويق إلى مواقع الطلب او الاستهلاك أو بعبارة أخرى فإن نموذج النقل هو خطة النقل لعدد من المنتجات (سلع أو خدمات) من عدد من مصادر الإتتاج أو التجهيز إلى عدد من مواقع الطلب أو الاستهلاك بأقل كلفة نقل ممكنة.

إن نموذج النقل يعتمد على الافتراضات الأساسية الآتية: 
• إن جميع المواد المنقولة بين المصادر ومناطق الطلب متجانسة (Homogeneous). • عدم وجود عوائق لنقل بين أي مصدر للتجهيز وأي موقع للطلب. إن مجموع كمية الطلب المتوفرة لدى المصدر يساوي مجموع كمية الطلب في المواقع. إن تكاليف نقل المواد بين أي مصدر وأي موقع للطلب معروفة ولن تتغير في الأمد القريب. إن كلفة النقل بين أي مصدر وأي موقع لن تتغير بتغير كمية المواد المنقولة. إن الهدف الرئيسي لمشكلة النقل هو تخفيض تكاليف النقل الكلية بين مصادر التجهيز ومناطق

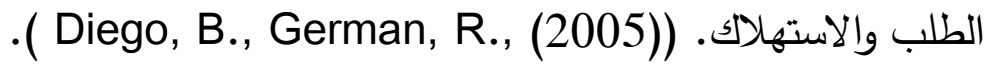
النموذج الرياضي لمشكلة النقل 1-نفترض أن عدد المصادر هو m ونفترض عدد مناطق الطلب هي n.

2-نفترض أن تكلفة نقل الوحدة الواحدة من المواد المنقولة من المصدر (i) الى منطقة الطلب ( ) ) وأن هذه الكلفة هي

3- إن كل مصدر يحتوي على كمية من البضاعة تصل إلى حد معين ولنفترض أن المصدر (i)

يحتوي على a $a_{i}$ 4- 4 نفترض أن الكمية المنقولة هي

5-ولتسهيل دراسة المشكلة وإيجاد الحلول لها نقوم بوضع مشكلة النقل على شكل جدول وهذا الجدول يسمى بجدول النقل حيث تتقسم إلى قسمين هما جدول التكاليف وجدول التوزيع حيث إن (Yih-Long, C., جدول التوزيع هو عبارة عن الكميات المنقولة من المصدر إلى منطقة الطلب (2001) أما جدول الكلفة فهو عبارة عن كلفة النقل من المصدر إلى منطقة الطلب. طرائق حل مشكلة النقل

هناك ثلاث طرق رئيسية لإيجاد الحل الأساسي لمشكلة النقل من (Yih-Long, C., (2001)) وهى: 1-طريقة الركن الثمالي الغربي (North west corner): تعد هذه الطريقة من أسهل الطرق لحل مشكلة النقل حيث تبدأ عملية إيجاد الحل الأساسي الأول من الزاوية الثمالية الغربية ولذلك سميت هذه الطريقة بهذا الاسم. 
2-طريقة أقل كلفة (The Least -Cost Method): يتم العمل بهذه الطريقة على أساس أقل

الكلف حيث يتم مشاهدة جدول التكاليف ومن ثم تخصيص الكمية المطلوبة على أساس أقل الكلف.

3-طريقة فوجل (Vogel Approximation Method): تعد هذه الطريقة من أفضل الطرق

وأدقها لما تتميز به هذه الطريقة من القدرة للوصول للحل الأمثل أو الحل القريب من الحل الأمثل ونقصد بالأفضلية الوصول إلى الحل الأمثل بأسرع وقت ممكن.

تحويل نموذج النقل إلى نموذج البرمجة الخطية

إن فكرة تحويل مشكلة النقل (تدنية تكاليف النقل) إلى نموذج برمجة خطية هي بالأساس تتم بتحويل مشكلة النقل بجملتها إلى دالة الهدف (OBJECTIVE FUNCTION) من نوع تصغير (CONSTRAINTS) وقيود (minimization) مشكلة برمجة خطية هو بالثكل الآتي:

$$
\begin{gathered}
\text { Min } z=\sum_{i=1}^{m} \sum_{j=1}^{n} \boldsymbol{C}_{i j} \boldsymbol{X}_{i j} \\
\sum_{j=1}^{n} x_{i j}=S_{i} i=1,2, \ldots . n \\
\sum_{i=1}^{m} x_{i j}=d_{j} j=1,2, \ldots m \\
x_{i j} \geq 0
\end{gathered}
$$

حيث إن X $x_{i j}$ هي الكمية المنقولة من المصدر i إلى المنطقة ز •.

Cي كلفة النقل من المصدر i إلى المنطقة ز

(David, R., Anderson, D., Sweeney, هي كمية التجهيز المتوفرة في المصدر $i$ .(J.,Tomas, A., (2001) وذلك باستخدام العلاقات الرياضية، سنقوم الآن بإيجاد الحل الأمثل لكمية السردين والتونة والثلج المجروش في تثاركية مرسى زواغة لتعليب وتسويق الأسماك بصبراتة. 


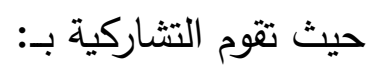

حفظ الأسماك ومنتجاتها بوسائل التجميد والتعليب وتصنيع مخلفات الأسماك وتحويلها إلى مساحيق تستخدم لأعلاف الحيوانات.

• استخراج زيوت الأسماك واستيراد الأسماك المجمدة وتعليبها وتسويقها وتصدير المعلبات

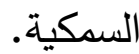

متغيرات القرار

م 2x $x_{2}$ كمية التونة المنتجة في المصنع لمدة يوم واحد بالطن.

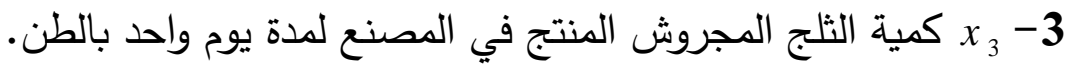

دالة الهدف اتضح من خلال الدراسة أن صافي الربح للسردين المعلب هو 1500 دينار، وأن صافي الربح للتونة المعلبة حوالي 1400 دينار، وصافي الربح للثلج المجروش حوالي 120 دينار وبالتالي تكون دالة الهدف على الصورة:

$$
P_{5}: 1500 x_{1}+1400 x_{2}+120 x_{3} \text { Maximise }
$$

القيود

أولاًِ- قيد الثلج: اتضح من الدراسة أن المصنع يصنع حوالي 4طن يومياً. وبالتالي يكون القيد على

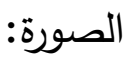

$$
x_{3} \leq 4
$$

ثانياً- قيد العمال: تبين من خلال الدراسة أن طن السردين يحتاج إلى 7 عمال لتصنيعه، و 4عمال لتصنيع طن التونة وأن عدد العمال الكلي 60 عاملا يومياً. وبالتالي يكون القيد على الثكل:

$$
7 x_{1}+4 x_{2} \leq 60
$$

ثالثاً- قيد زيت الزيتون: تبين من الدراسة أن الطن الواحد من السردين يحتاج إلى 160 لتر من الزيت، والطن الواحد من التونة يحتاج إلى 250لتر من الزيت وأن كمية الزيت المستخدمة يومياً للإنتاج 2000 لتز وعليه يكون القيد كالتالي: 


$$
160 x_{1}+250 x_{2} \leq 2000
$$

رابعاً- قيد الخام من التن والسردين: الخام المتوفر للسردين 0.850طن، والخام للتونة 0.9طن والكمية الكلية المتوفرة 6.5طن وبالتاي يكون القيد كالتالي:

$$
0.850 x_{1}+0.9 x_{2} \leq 6.5
$$

خامساً- قيد الحد الأدنى للإنتاج: الحد الأدنى لكل من السردين والتونة حوالي 1.5طن وبالتالي القيود هي:

$$
\begin{aligned}
& x_{1} \succ 2.5 \\
& x_{2} \succ 2.5
\end{aligned}
$$

$$
P_{5}: 1500 x_{1}+1400 x_{2}+120 x_{3} \quad \text { Maximise }
$$

subject to

$$
\begin{gathered}
x_{3} \leq 4 \\
7 x_{1}+4 x_{2} \leq 60 \\
160 x_{1}+250 x_{2} \leq 2000 \\
0.850 x_{1}+0.9 x_{2} \leq 6.5 \\
x_{1} \succ 2.5 \\
x_{2} \succ 2.5 \\
x_{1}, x_{2}, x_{3} \geq 0
\end{gathered}
$$

حل مشاكل البرمجة الخطية باستخدام (Lindo)

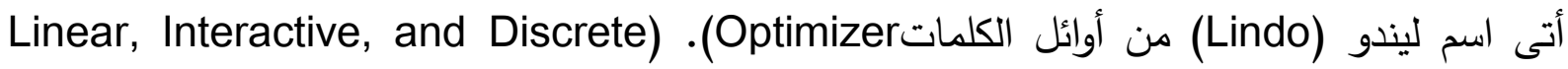
وهو يعد من أشهر وأقوى البرامج المتخصصة في حل مشاكل البرمجة الخطيه وغير الخطيه . وما يميز هذا البرنامج هو سهولة الاستخدام حيث يمكن نسخ المشكلة بالثكل المعتاد وبالصياغة الرياضية المناسبة ولصقها في نافذة البرنامج أو يمكن كتابتها مباشرة على نافذة البرنامج كما تكتب في

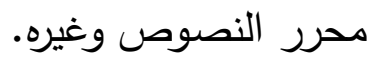


وقد تم استخدام برنامج LINDO لإيجاد الحل الأمثل لهذه المشكلة وقد اتضح أن الحل الأمثل الذي يمكن التثاركية من تحقيق أقصي ربح هو كالتالي:

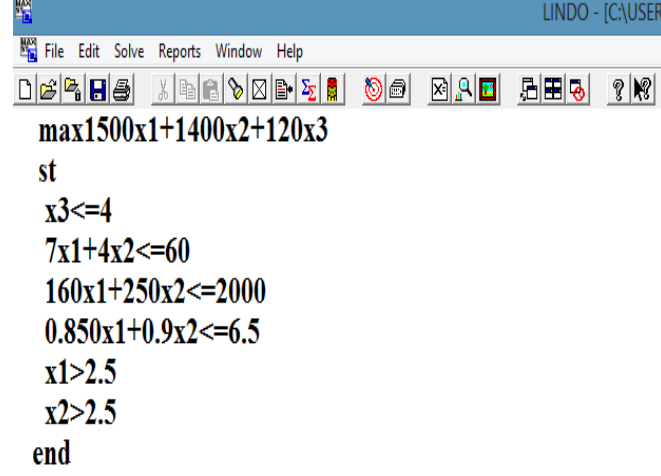

شكل (1): تطبيق المثال في برنامج LINDO.

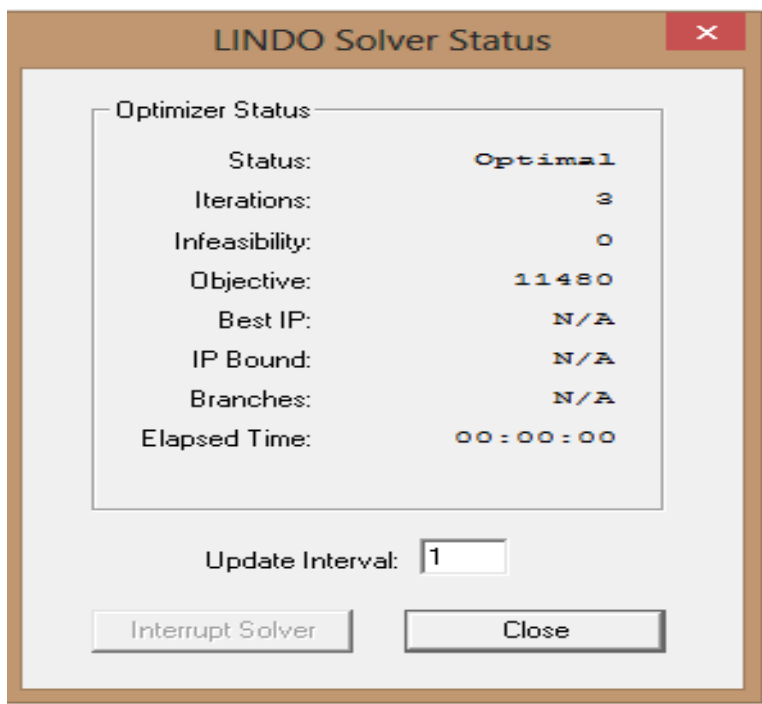

شكل (2): نتائج برنامج LINDO. 


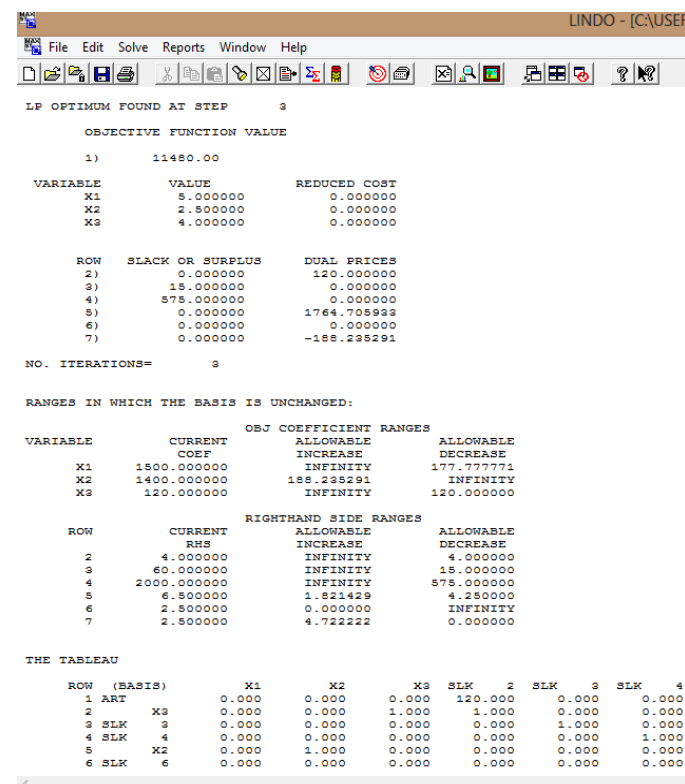

شكل (3): نتائج برنامج LINDO.

حل مشاكل البرمجة الخطية باستخدام برنامج WINQSB يعد برنامج WINQSB من أكثر البرامج تخصصا في البرمجه الخطيه والذي يستخدم في تطبيقات بحوث العمليات والبرمجة الخطية ويعطي نتائج مرضية.

\begin{tabular}{|c|c|c|c|c|c|c|c|}
\hline \multicolumn{8}{|c|}{ 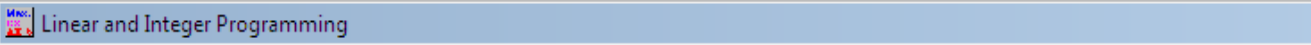 } \\
\hline \multicolumn{8}{|c|}{ File Edit Format Solve and Analyze Results Utilities Window WinQSB Help } \\
\hline 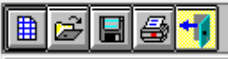 & 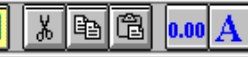 & 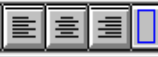 & $\Leftrightarrow \Leftrightarrow$ & $D$ & 四 & 2) & \\
\hline \multicolumn{8}{|l|}{ 3. MAX } \\
\hline \multicolumn{8}{|c|}{ C6 : R.H.S. $\quad x \vee 2.5$} \\
\hline & & Variable --> & $\mathrm{X} 1$ & $\times 2$ & $\times 3$ & Direction & R. H. S. \\
\hline & & Maximize & 1500 & 1400 & 150 & & \\
\hline & & C1 & & & 1 & $<=$ & 4 \\
\hline & & C2 & 7 & 4 & & $<=$ & 60 \\
\hline & & C3 & 160 & 250 & & $<=$ & 2000 \\
\hline & & C4 & 0.850 & 0.9 & & $<=$ & 6.5 \\
\hline & & C5 & 1 & & & \rangle$=$ & 2.5 \\
\hline & & C6 & & 1 & & \rangle$=$ & 2.5 \\
\hline & & LowerBound & $\mathbf{0}$ & $\mathbf{0}$ & $\mathbf{0}$ & & \\
\hline & & UpperBound & M & M & M & & \\
\hline & & VariableType & Continuous & Continuous & Continuous & & \\
\hline
\end{tabular}

شكل (4): تطبيق المثال في برنامج WINQSB. 


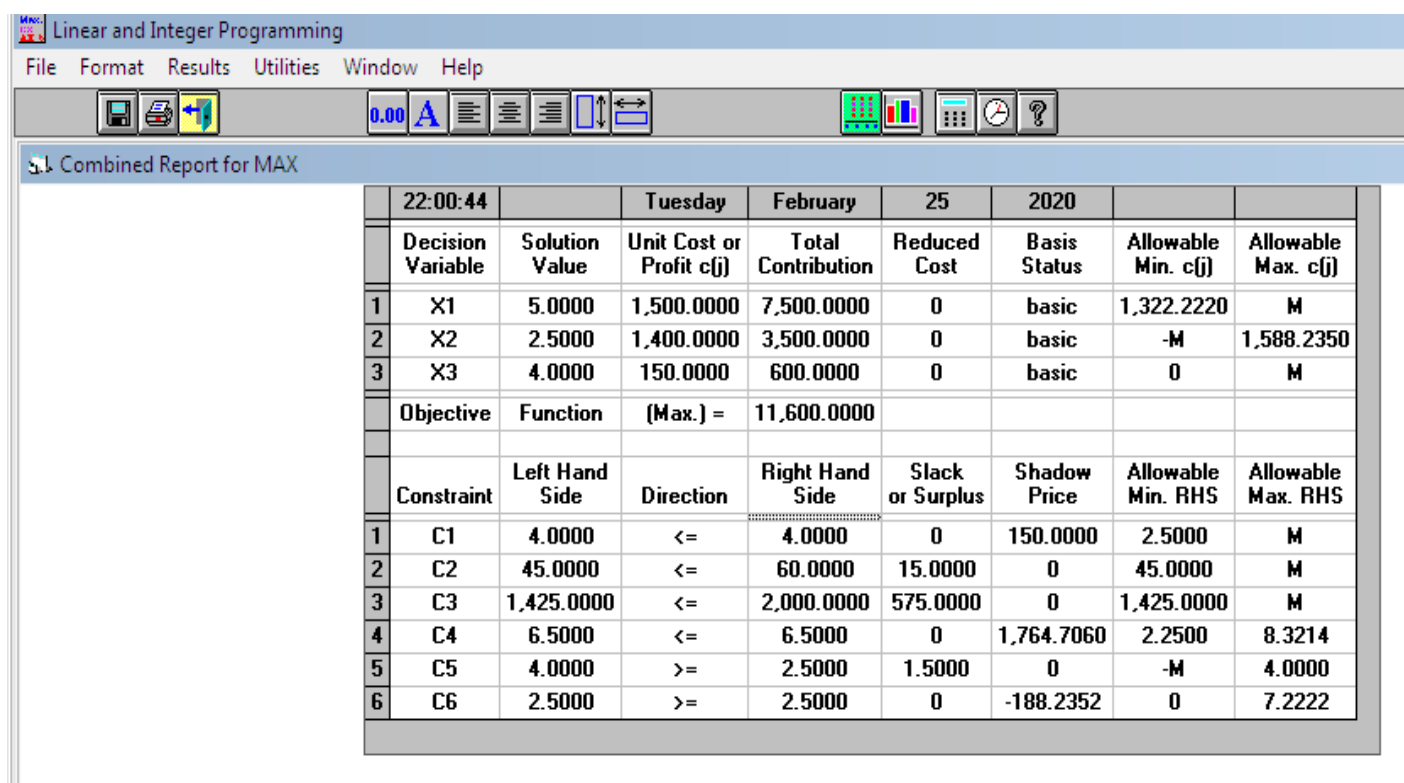

\section{شكل (5): تطبيق المثال في برنامج WINQSB.}

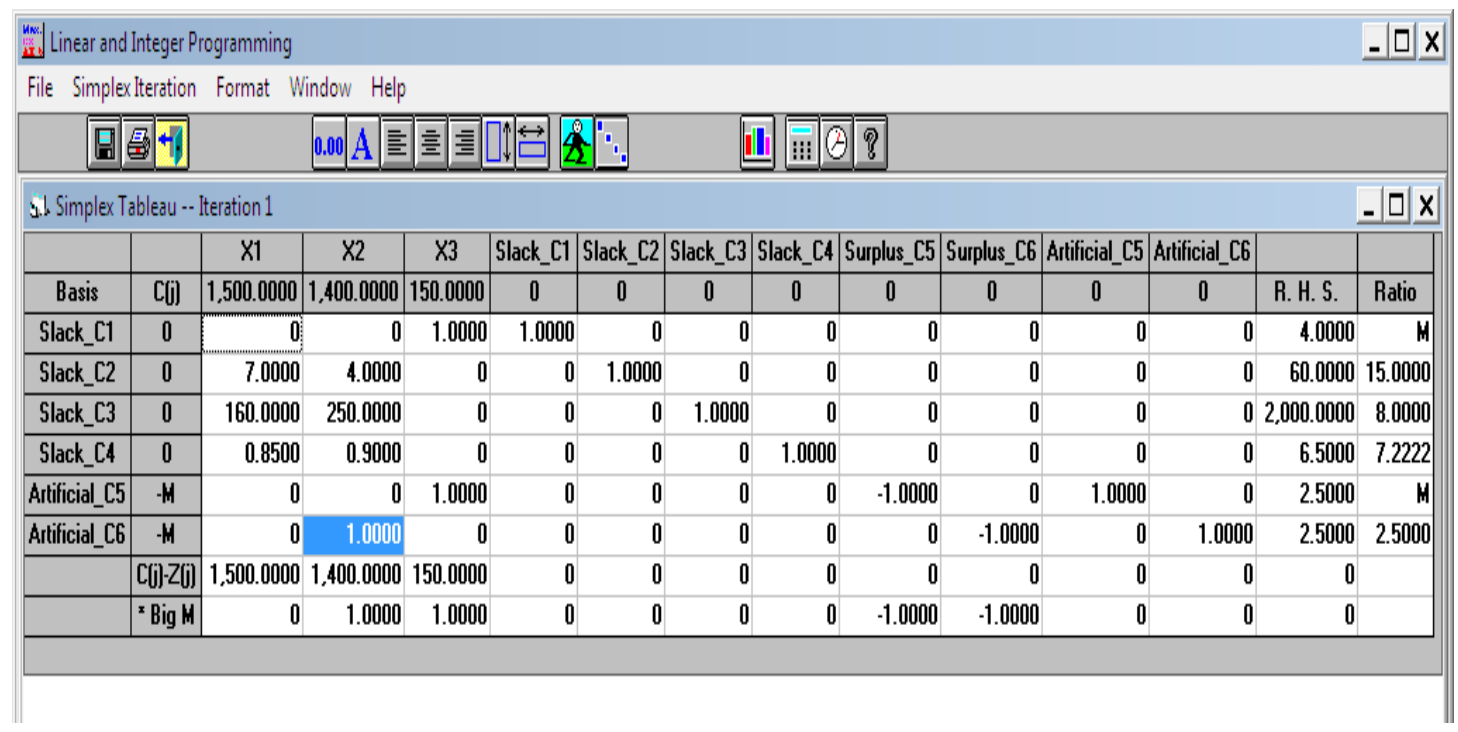

شكل (6): نتائج برنامج WINQSB 


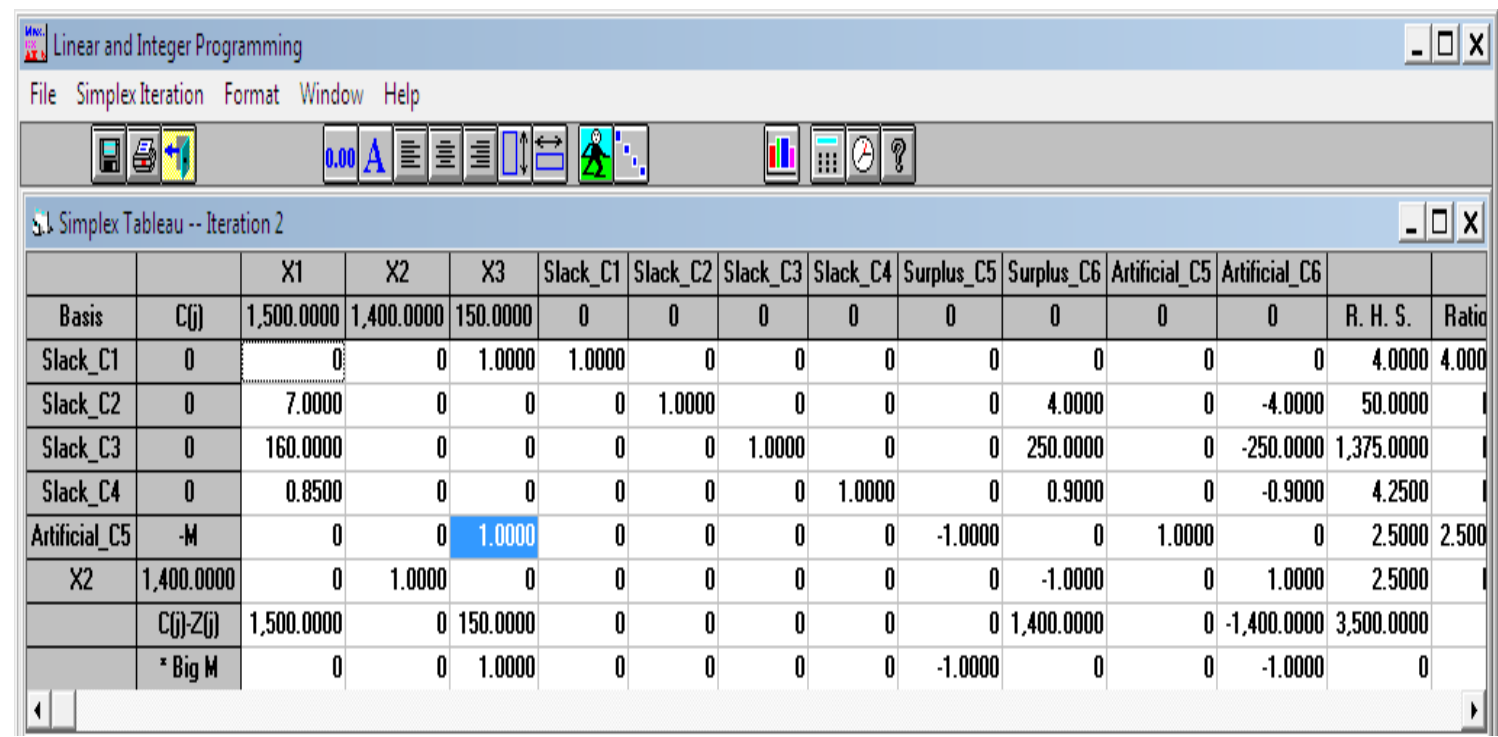

\section{شكل (7): نتائج برنامج WINQSB.}

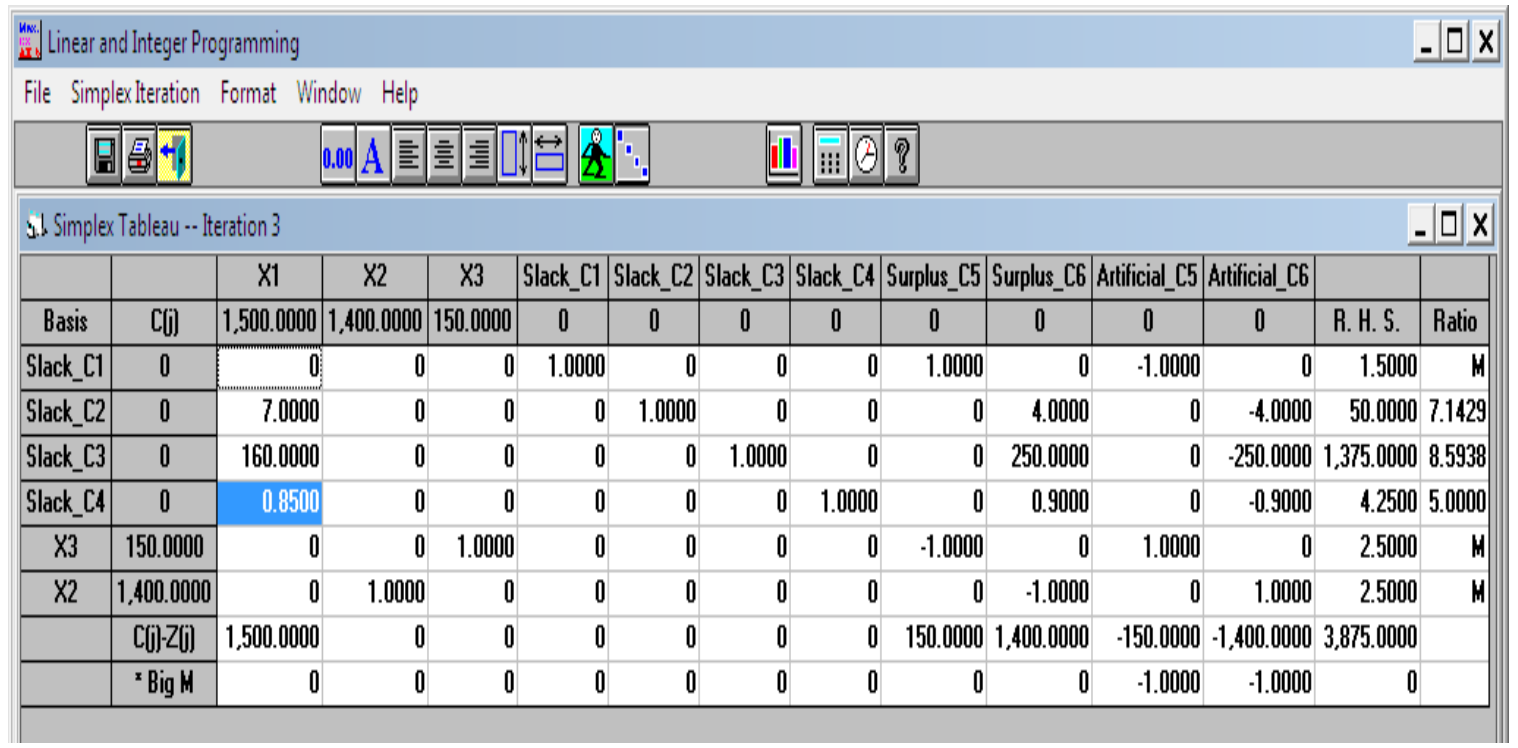




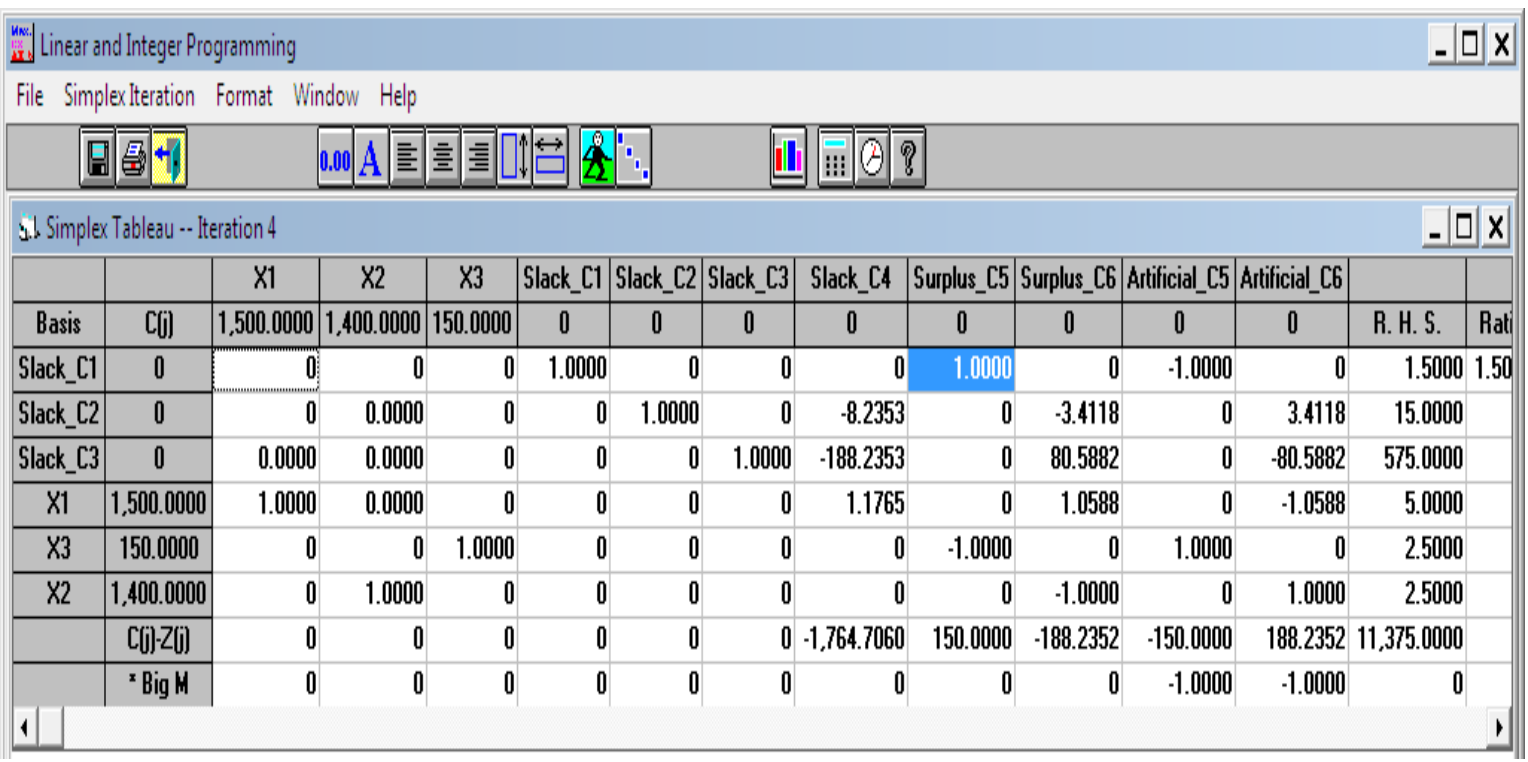

\section{شكل (9): نتائج برنامج WINQSB.}

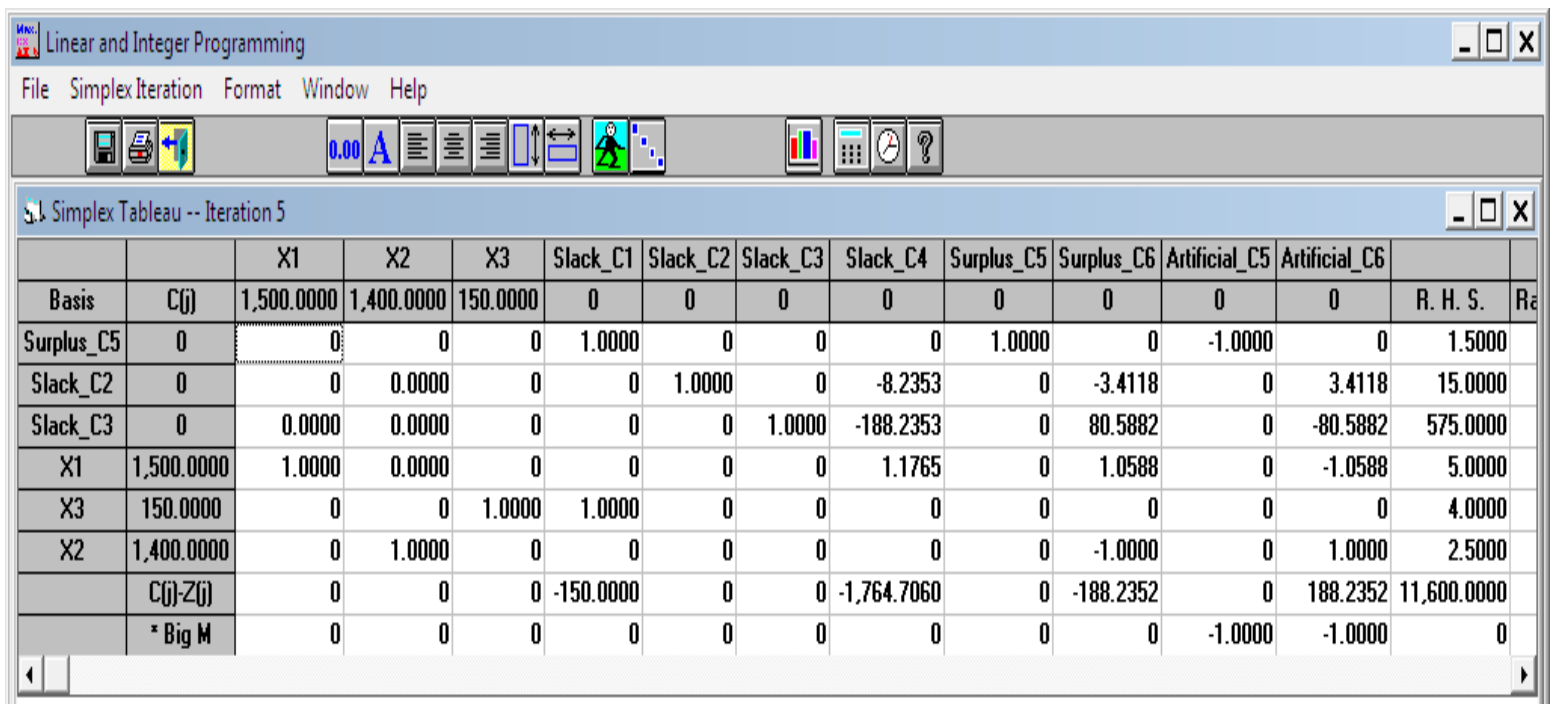

شكل (10): نتائج برنامج WINQSB. 


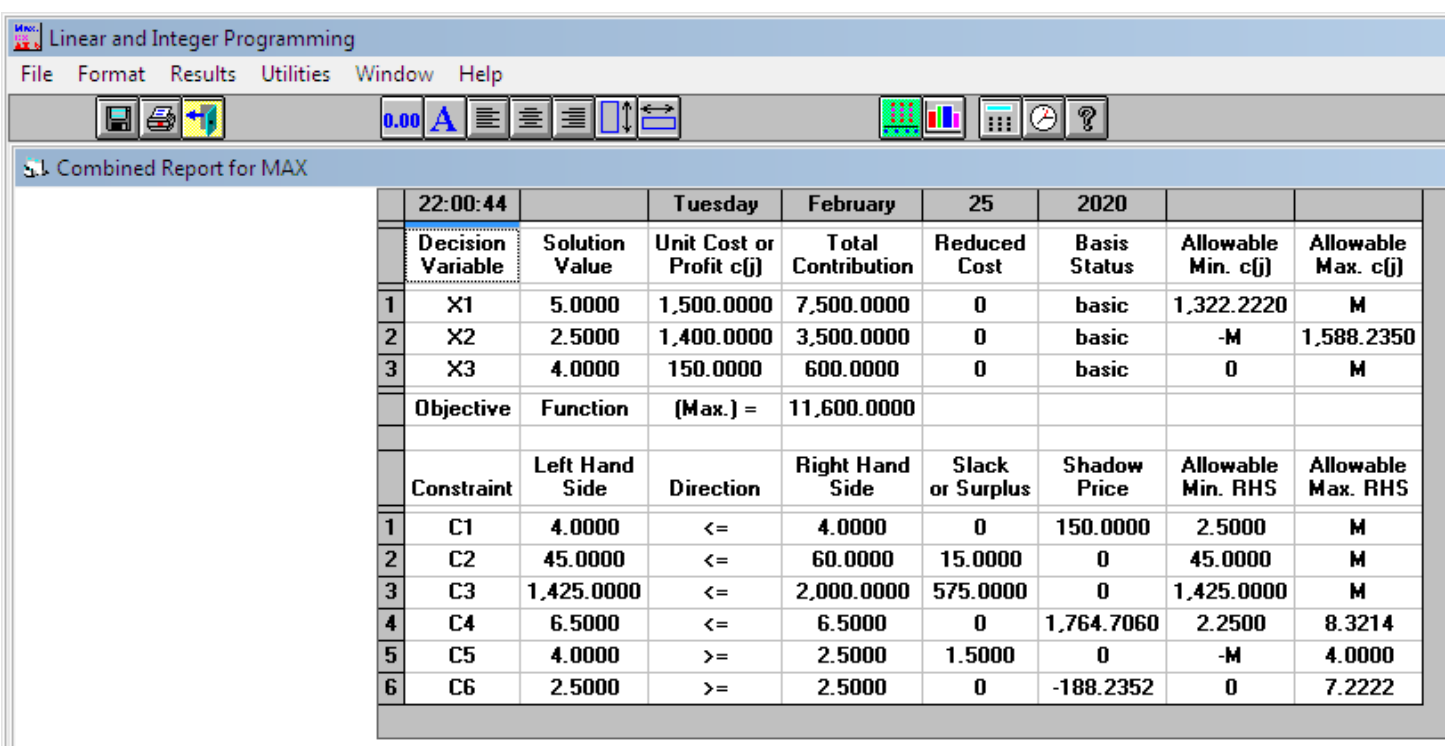

\section{شكل (11): النتائج الاخيره لبرنامج WINQSB.}

\section{جدول (1): النوع المنتج والكمية والربح}

\begin{tabular}{|c|c|c|}
\hline النوع & الكمية & الربح بالدينار \\
\hline السردين & 5طن & \multirow{3}{*}{11480} \\
\hline التونة & 2.5طن & \\
\hline الثلج المجروش & 4طن & \\
\hline
\end{tabular}

والآن سنقوم بالحل باستخدام طريقة السمبلكس ذات المرحلتين ويكون الحل على الصورة:-

$$
P_{5}: 1500 x_{1}+1400 x_{2}+120 x_{3} \text { Maximise }
$$

subject to

$$
\begin{gathered}
7 x_{1}+4 x_{2} \leq 60 \\
160 x_{1}+250 x_{2} \leq 2000 \\
0.850 x_{1}+0.9 x_{2} \leq 6.5 \\
x_{3} \leq 4
\end{gathered}
$$




$$
\begin{gathered}
x_{1} \succ 2.5 \\
x_{2} \succ 2.5 \\
x_{1}, x_{2}, x_{3} \geq 0 \\
: \text { بوضع المسألة في شكلها القانوني تكون على الصورة } \\
P_{5}: 1500 x_{1}+1400 x_{2}+120 x_{3} \text { Maximise }
\end{gathered}
$$

subject to

$$
\begin{gathered}
7 x_{1}+4 x_{2}+s_{1}=60 \\
160 x_{1}+250 x_{2}+s_{2}=2000 \\
0.850 x_{1}+0.9 x_{2}+s_{3}=6.5 \\
x_{3}+s_{4}=4 \\
-10 x_{1}+s_{5}=-25 \\
-10 x_{2}+s_{6}=-25 \\
x_{1}, x_{2}, x_{3}, s_{1}, s_{2}, s_{3}, s_{4}, s_{5}, s_{6} \geq 0
\end{gathered}
$$

جدول (2): يوضح جدولة البيانات بطريقة السمبلكس ذات المرحلتين

\begin{tabular}{|l|l|c|l|l|}
\hline$P_{5}$ & $x_{1}$ & $x_{2}$ & $x_{3}$ & $T_{1}$ \\
\hline$s_{1}$ & 7 & 4 & 0 & 60 \\
\hline$s_{2}$ & 160 & 250 & 0 & 2000 \\
\hline$s_{3}$ & 850 & $(900)$ & 0 & 6500 \\
\hline$s_{4}$ & 0 & 0 & 1 & 4 \\
\hline$s_{5}$ & -10 & 0 & 0 & -25 \\
\hline$s_{6}$ & 0 & -10 & 0 & -25 \\
\hline$z^{I}$ & -10 & -10 & 0 & -50 \\
\hline
\end{tabular}


جدول (3): يوضح خطوات طريقة السمبلكس ذات المرحلتين لعمليات المحورة لجدول (2)

\begin{tabular}{|c|c|c|c|c|}
\hline$P_{5}$ & $x_{1}$ & $s_{3}$ & $x_{3}$ & $T_{2}$ \\
\hline$s_{1}$ & $29 / 9$ & $-4 / 900$ & 0 & $280 / 9$ \\
\hline$s_{2}$ & $-685 / 9$ & $-25 / 90$ & 0 & $1750 / 9$ \\
\hline$x_{2}$ & $85 / 90$ & $1 / 900$ & 0 & $65 / 9$ \\
\hline$s_{4}$ & 0 & 0 & 1 & 4 \\
\hline$s_{5}$ & -10 & 0 & 0 & -25 \\
\hline$s_{6}$ & $(85 / 9)$ & $1 / 90$ & 0 & $425 / 9$ \\
\hline$z^{I}$ & -10 & 0 & 0 & -25 \\
\hline
\end{tabular}

جدول (4): يوضح خطوات طريقة السمبلكس ذات المرحلتين لعمليات المحورة لجدول (3)

\begin{tabular}{|c|c|c|c|c|}
\hline$P_{5}$ & $s_{6}$ & $s_{3}$ & $x_{3}$ & $T_{3}$ \\
\hline$s_{1}$ & $-29 / 85$ & $-7 / 850$ & 0 & $225 / 17$ \\
\hline$s_{2}$ & $685 / 85$ & $-30 / 153$ & 0 & $9775 / 17$ \\
\hline$x_{2}$ & $-1 / 10$ & 0 & 0 & $425 / 170$ \\
\hline$s_{4}$ & 0 & 0 & $(1)$ & 4 \\
\hline$s_{5}$ & $90 / 85$ & $1 / 85$ & 0 & $425 / 17$ \\
\hline$x_{1}$ & $9 / 85$ & $1 / 850$ & 0 & 5 \\
\hline$z^{I}$ & 0 & 0 & 0 & 0 \\
\hline$z$ & $320 / 17$ & $30 / 17$ & -120 & $187000 / 17$ \\
\hline
\end{tabular}


جدول (5): يوضح خطوات طريقة السمبلكس ذات المرحلتين لعمليات المحورة لجدول (4)

\begin{tabular}{|c|c|c|c|c|}
\hline$P_{5}$ & $s_{6}$ & $s_{3}$ & $s_{4}$ & $T_{4}$ \\
\hline$s_{1}$ & $-29 / 85$ & $-7 / 850$ & 0 & $225 / 17$ \\
\hline$s_{2}$ & $685 / 85$ & $-30 / 153$ & 0 & $9775 / 17$ \\
\hline$x_{2}$ & $-1 / 10$ & 0 & 0 & $425 / 170$ \\
\hline$x_{3}$ & 0 & 0 & 1 & 4 \\
\hline$s_{5}$ & $90 / 85$ & $1 / 85$ & 0 & $425 / 17$ \\
\hline$x_{1}$ & $9 / 85$ & $1 / 850$ & 0 & 5 \\
\hline$z$ & $320 / 17$ & $30 / 17$ & 120 & $195160 / 17=11.480$ \\
\hline
\end{tabular}

$$
z^{*}=11.480 \text { وقيمة دالة الهحف هي }\left(x_{1}^{\wedge}, x_{2}^{\wedge}, x_{3}^{\wedge}\right)=(5,2.5,4)
$$

j $d_{j}$

1-لو نظرنا الى الثكل (2) لوجدنا انها نفس النتائج التي تحصلنا عليها في الجدول (5) وهي النتائج المثلى التي تحصلنا عليها بعد استخدام طريقة السمبلكس ذات المرحلتين وبرنامج الليندو بينما باستخدام برنامج WINQSB تكون قريبه جدا بقيمه 11600 اتقريبا. 2-سهولة الحل باستخدام برنامج الليندو وبرنامج وينكس، حيث يوفر لنا الوقت والجهد لحل مسالة

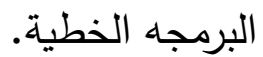
3- أظهرت نتائج البحث أرباح الشركة للكميات المدروسة بقيمة 11480 دينار تقريبا. 
أ. رند عمران مصطفى الأسطل (2016م). بحوث العمليات والأساليب الكمية في صنع القرارات الاداريه الناشر مكتبة الطالب الجامعي، جامعة الأقصى، فلسطين، الطبعة السادسة.

• عمر محمد ناصر حسين، عبيد محمود نصر الزوبعي، عادل موسى يونس، (2012م). تطبيقات

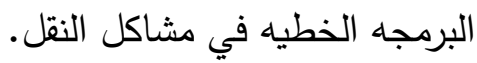

ا.د.حسين عطا غنيم، بحوث العمليات، جامعة القاهرة.

ا.د. حلمي عبدالفتاح الثبيثي، د. سيد عبدالعاطي، د. طه الطاهر ابراهيم اسماعيل، (1993م)، بحوث العمليات في المحاسبه، جامعة القاهرة.

ه. دميد ناصر الفتال، د. دلال صادق الجواد، (2008م). بحوث العمليات، دار اليلزوي للنشر، عمان.

ه.زيد تميم البلخي، (1998م). مقدمه في البحوث العمليات، الطبعه الاولى، الرياض، جامعة الملك

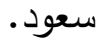
ا.د. محمد عبدالعال النعيمي واخرون، (1999م). دار وائل، الطبعه الاولى. ه. دحمد محمد كعبور، (2005م). اساسيات بحوث العمليات نماذج وتطبيقات، منشورات اكادمية

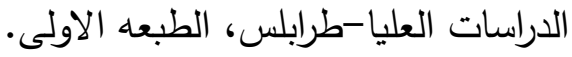

• النجار ظافر حين النجار،صباح كريمالقيسي.ثائر فيصل .(2009). الاساليب الكميه في الادارة.مطبعة جامعة بغداد.بغداد_العراق،عدد الصفحات 387.

2- المراجع الاجنبية:

- Hillier, T., Liberman, J., (2005). Introduction to the Operation Research, McGraw-Hill, USA. 
- Diego, B., German, R., (2005). Linear programming solvers for Markov decision processes, McGraw -Hill, U. S. A.

- David, R., Anderson, D., Sweeney, J.,Tomas, A., (2001). Quantitative Methods for Busines,. South -Western Colleg,. India._

- Yih-Long, C., (2001). WinQsb, Jon Willey and Sons, U. S. A. 